\title{
PARAMETERS FOR LOW ENERGY OPERATION OF CESR*
}

\author{
D. Rice ${ }^{\dagger}$, G. Codner, G. Dugan, R. Ehrlich, Z. Greenwald, Y.He, S. Henderson Y. Li, \\ V. Mejidzade, A. Mikhailichenko, N. Mistry, E. Nordberg, J. Rogers, \\ D. Rubin, D. Sagan, E. Smith, A. Temnykh, M. Tigner, Cornell University, Ithaca, NY 14853, USA
}

\begin{abstract}
We present a detailed design for operation of the Cornell Electron Storage Ring, CESR, in the energy range covering the $\Psi$ resonances and the Charm and Tau thresholds, as well as reaching to the upper $\mathrm{Y}$ resonances near $11 \mathrm{GeV}$. The addition of $18 \mathrm{~m}$ of super-ferric wiggler magnets will partially restore low energy beam emittance and damping times. Installation of superconducting quadrupoles in the interaction region and the addition of high performance superconducting RF cavities will enhance performance at all energies. Studies of optics, beam dynamics, wiggler and vacuum system performance, beam stability, and beam-beam effects confirm operation with a luminosity of $3 \times 10^{32} \mathrm{~cm}^{-2}-\mathrm{sec}^{-1}$ at $1.88 \mathrm{GeV}$.
\end{abstract}

\section{INTRODUCTION}

\subsection{The Development of CESR}

CESR is a $4.7-6 \mathrm{GeV} / \mathrm{beam} \mathrm{e}^{+} \mathrm{e}^{-}$collider that has been operating at the $\mathrm{B}$ meson threshold with peak luminosity well above $10^{33} \mathrm{~cm}^{-2}-\mathrm{sec}^{-1}$, and a consistent integrated luminosity per month of $1.4 \mathrm{fb}^{-1}$ [1]. This performance level has been reached by incorporating innovative ideas in a series of upgrades to the operation of CESR.

Forty-five bunches in each beam circulate in a single vacuum chamber and collide at a single interaction point in the middle of the CLEO detector. Super-conducting $\mathrm{RF}$ cavities provide the required $\mathrm{RF}$ voltage while introducing minimal parasitic mode impedance to the ring. Permanent magnet IR quadrupoles minimize the chromaticity generated in optics with $\beta^{*}{ }_{\mathrm{V}}$ of $1.8 \mathrm{~cm}$.

Continuing this program of innovative upgrades will extend the operating range of CESR to the $J / \Psi$ $\left(\mathrm{E}_{\text {beam }}=1.55 \mathrm{GeV}\right)$ through the $\mathrm{Y}$ resonances $\left(\mathrm{E}_{\text {beam }} \approx 6\right.$ $\mathrm{Gev})$. The luminosity performance will provide factors $>10$ increase in present world Charm data sets in a few years of running.

\subsection{Charm Physics at CESR/CLEO}

The Charm/Tau regime is excellent ground for studies of weak interaction physics and tests of QCD. A data sample 10-20 times that accumulated to date, combined with the excellent resolution and hermiticity of the CLEO detector will improve by 5-15 the precision of branching ratios and decay constants. Comparison with CLEO data on the $\mathrm{Y}$ resonances will be used in searching for glue-

\footnotetext{
* Work supported by US National Science Foundation

${ }^{\dagger}$ mailto:dhr1@ cornell.edu
}

rich states. These measurements will be much cleaner than comparable measurements from the B factories.

\section{TOWARD LOWER ENERGY}

\subsection{Conventional Collider Energy Scaling}

Experience with $\mathrm{e}^{+} \mathrm{e}^{-}$colliders accumulated over many years suggests that in a given machine the peak luminosity scales as the $4^{\text {th }}$ power of the beam energy. The components of this scaling are revealed in the following equations for luminosity and horizontal beambeam parameter:

$$
\begin{gathered}
L=2.17 \times 10^{32}(1+r) \frac{n_{b} i_{b} E_{0} \xi_{y}}{\beta_{y}^{*}} \\
\xi_{x}=4.77 \times 10^{-9} \frac{C_{L} i_{b}}{E_{0} \varepsilon_{x}(1+r)}
\end{gathered}
$$

where $L\left(\mathrm{~cm}^{-2}-\mathrm{sec}^{-1}\right)$ is luminosity, $r$ the beam aspect ratio $(\mathrm{y} / \mathrm{x})$ at the interaction point, $n_{b}$ the number of bunches per beam, $i_{b}(\mathrm{~A})$ the bunch current, $E_{0}(\mathrm{GeV})$ the beam energy, $\xi_{y, x}$ the beam-beam space charge parameter (vertical, horizontal), $\beta_{y}{ }^{*}$ the vertical focusing function at the i.p., $C_{L}(\mathrm{~m})$ the circumference of the machine and $\varepsilon_{x}$ (m-rad) the horizontal emittance.

Combining these two equations with the condition that $\mathrm{i}_{\mathrm{b}}$ is limited by $\xi_{x}$ yields:

$$
L=4.55 \times 10^{40}(1+r)^{2} \frac{n_{b} E_{0}^{2} \xi_{y} \xi_{x} \varepsilon_{x}}{\beta_{y}^{*} C_{L}}
$$

We can see that even if all other parameters are constant, luminosity scales as $E_{0}^{2}$. In practice $\xi_{y}, \xi_{x}$ and $\varepsilon_{x}$ often vary with energy, producing a steeper scaling law.

\subsection{Improved Energy Scaling}

$\xi_{y}, \xi_{x}$, and particularly $\varepsilon_{x}$ are influenced by synchrotron radiation effects. $\varepsilon_{x}$ is determined by optics parameters and synchrotron radiation spectrum [2], and the beambeam parameters, $\xi$, empirically scale $\propto E_{-}^{0 \rightarrow 1}$ where the exponent is usually closer to one than zero. With careful manipulation of radiation effects, one should achieve a scaling closer to $L \propto E_{0}^{2}$.

Maximum achievable values of the beam-beam parameters, $\xi_{y, x}$, are likely related to radiation damping's cooling effect on the transverse and longitudinal "temperature" of the beams, which are heated by nonlinear beam-beam driven resonances. The frequently found scaling of $\xi \propto \mathrm{E}_{0}$ in a given ring corresponds to $\xi \propto$ $\tau^{-1 / 3}$ ( $\tau=$ damping time). 
Wiggler magnets can provide controllable radiation without large effects on beam orbits. When the wigglers dominate radiation in a ring, the following scaling applies:

$$
\begin{array}{ll}
\text { Damping time: } & \tau \propto \frac{1}{L_{W} B_{W}^{2}} \\
\text { Horizontal Emittance: } & \varepsilon_{X} \propto B_{W} H_{W} \\
\text { Energy spread: } & \frac{\sigma_{E}}{E_{0}} \propto B_{W}
\end{array}
$$

New variables are $L_{W}$, the length of wigglers; $B_{W}$, the peak magnetic field in the wigglers; $H_{W}$, the square of the normalized dispersion at the wiggler, and $\sigma_{E}$ the beam's R.M.S. energy spread.

We immediately see that the peak field of the wiggler is limited by the maximum acceptable energy spread, and the total length of the wigglers is then determined by the desired damping time. The horizontal emittance may then be set by controlling $H_{W}$.

At $1.88 \mathrm{GeV}$ in CESR, $18.2 \mathrm{~m}$ of wigglers with 2.1 Tesla peak field will provide a $50 \mathrm{~ms}$ damping time (vs $20 \mathrm{~ms}$ at $5.3 \mathrm{GeV}$ ), the same emittance as at $5.3 \mathrm{GeV}$, and a relative energy spread of $8 \times 10^{-4}$.

\subsection{Other Factors}

The discussion on low energy performance of course assumes no other phenomenon limits performance. Critical issues include: single beam instabilities, beam lifetime, parasitic beam-beam effects, larger rotation angle in the experimental solenoid, the optics properties of the machine - both linear and non-linear, and performance of accelerator components.

\section{CESR-c}

The primary modifications to CESR in preparation for low energy running are:

- $\quad$ Replace the present permanent magnet IR quads with superconducting quads to expand operating energy range and lower $\beta$ at the interaction point.

- Upgrade RF cavity complement to shorten the bunch length compatible with lower $\beta^{*}$.

- Install $\sim 18 \mathrm{~m}$ of 2.1 Tesla wiggler magnets to enhance synchrotron radiation effects

The first two of these modifications have been in progress to improve operation at $5.3 \mathrm{GeV}$. The wiggler magnets are the only major upgrade specifically for Charm operation of CESR.

\subsection{Wiggler Magnets}

The required wiggler field $(2.1 \mathrm{~T})$ and the large pole gap $(>5.5 \mathrm{~cm}$ to avoid vertical aperture restrictions) eliminate permanent magnet (marginal field strength, weight) and normal conducting (power requirements) magnets. The CESR-c wiggler design [3] is based on superferric technology. Figure 1 shows a 3-pole test model.

The final magnets will be built in standard units, each with $1.3 \mathrm{~m}$ active length and a wiggler period of $\sim 40 \mathrm{~cm}$. The cryostats will have a warm bore, water cooled vacuum chamber and have a flange-to-flange length of
$1.7 \mathrm{~m}$ including a NEG vacuum pump port. Figure 2 shows a perspective view of the cryostat with cold mass.

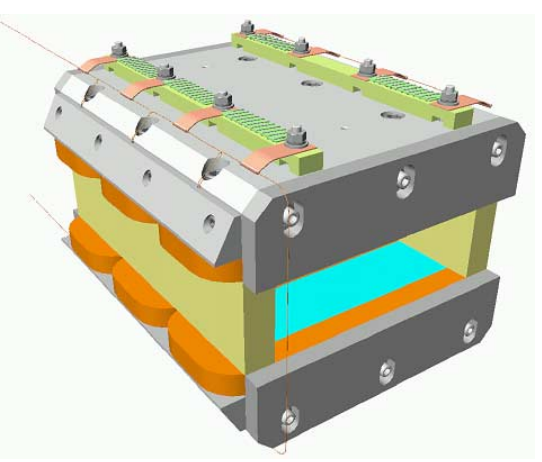

Figure 1: 3-pole test superferric wiggler cold mass

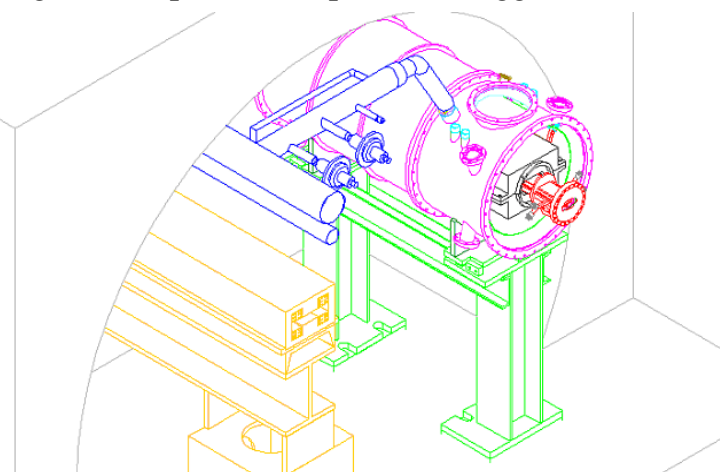

Figure 2: Full superferric wiggler and cryostat in tunnel

The wigglers will be placed in 4 groups symmetrically located in one third of the ring circumference close to utilities. This compact grouping reduces the length of transfer lines required and also facilitates the design of optics around the wigglers. 14 units will be required.

The cryogens will be provided through transfer lines extended from cryogen distribution for the present RF system. The three 600 watt existing refrigerators have ample capacity for the approximately 100 watt additional load from the wiggler cryostats and transfer lines.

While the effects of wiggler magnets on beam dynamics are small compared to their internal bending angles, CESR-c will be particularly sensitive to any nonlinear effects because of the low energy, the total length of wigglers, and the "pretzel" orbit used to separate beams at parasitic crossing points.

There are two sources of systematic nonlinearities from the wigglers. The first, present even in an ideal wiggler, arises from the beam passing at an angle through the longitudinal field between poles. The effective horizontal field seen by the beam is [4]:

$$
\int_{\text {wiggler }} B_{\chi} d s=-\frac{L_{W} B_{W}^{2}}{2 B \rho}\left(y+\frac{2}{3} k^{2} y^{3}+\ldots\right)
$$

where $B_{\chi}$ is the apparent horizontal field seen by the beam, $s$ is along the beam path, $B \rho$ the magnetic rigidity of the beam, and $\mathrm{k}$ is $2 \pi / \lambda_{W}$, where $\lambda_{W}$ is the wiggler period (twice pole length). This focusing is in the vertical plane only and has a linear part that is independent of $\lambda_{W}$ and an octupole like component that increases as $\lambda_{W}{ }^{-2}$. (Higher 
order terms are ignored here.) The linear focusing term causes a tune shift of 0.1 integer for each wiggler in CESR-c and must be designed into the optics.

A realistic wiggler field has a finite roll off across the pole face $\left(\Delta B_{y}(x)\right)$ and, combined with the beam's systematic displacement in the horizontal plane, creates an effective vertical field integral: [5]

$$
\int_{\text {Wiggler }} B_{y} d s=-\frac{1}{2} L_{W} A_{x} \frac{d B_{y}}{d x}
$$

Here $A_{x}$ is the peak amplitude of the beam wiggle (proportional to $\lambda_{W}{ }^{2}$ ). Unlike the vertical effects in equation 4 , these nonlinearities resemble conventional multipoles and they increase as $\lambda_{W}{ }^{2}$.

Incorporating many strong wigglers in the ring while maintaining good dynamic aperture requires careful design, optimizing $\lambda_{W}$ and field uniformity. A tracking code [6] has been used to model the wiggler nonlinearities. To date lattice designs have marginally acceptable dynamic aperture $\left(\sim 10 \sigma_{x, y}\right.$ with $4 \sigma_{\mathrm{E}} / \mathrm{E}$ energy deviation $)$ at 1.88 $\mathrm{GeV}$. We are working on further optimization of optics and wigglers, as well as octupole compensation to increase dynamic aperture to a conservative margin.

\subsection{Performance issues}

The previously mentioned superconducting RF and IR quadrupoles are described elsewhere in this conference $[7,8]$.

Vacuum pumping: The distributed sputter ion vacuum pumps in the CESR arcs use the dipole magnet fields. At $1.88 \mathrm{GeV}$ their pumping speed will drop to near zero. Experiments have shown that the chamber walls will adsorb any released gasses, keeping average pressures below 1 ntorr at CESR-c design currents. Occasional running at $5 \mathrm{GeV}$ (planned for synchrotron radiation users) will clean up adsorbed gases from vacuum interventions.

Injection: The linac and synchrotron injector for CESR have recently operated with $1.8 \mathrm{GeV}$ electron and positron beams which have been taken through transport lines up to the CESR vacuum system. The present repetition rate of $60 \mathrm{~Hz}$ will be reduced (by approximately the ratio of damping times at 1.88 and $5 \mathrm{GeV}$ ) to 20 or $30 \mathrm{~Hz}$. Otherwise injection will be similar to that at $5.3 \mathrm{GeV}$.

Beam Stability: The transverse and longitudinal feedback systems will provide increased kicks at lower energy to match the increased effects of parasitic impedances. For potential quadrupole and higher modes, the damping time increases $\sim 2.5 \mathrm{x}$ and currents decrease $\sim 2 \mathrm{x}$, providing adequate damping for instabilities with thresholds at least $25 \%$ above $5.3 \mathrm{GeV}$ operating current.

Parasitic beam-beam effects: As with the instabilities, the decrease in current will substantially compensate the increased sensitivity of the beams to the parasitic crossing. There is also the possibility of compensating some of the bunch-by-bunch dipole kicks and tune shifts from the parasitic crossings using the feedback systems [9] and RF quadrupoles.

Beam lifetime: The single beam particle losses from beam-gas and Touschek scattering have been calculated and net lifetime varies from 4.0 to 5.3 hours between 1.55 and $1.88 \mathrm{GeV}$. With beam-beam losses initial lifetimes will be between 2 and 3 hours.

Experiment solenoid: The CLEO solenoid will be decreased in strength from 1.5 to 1.0 Tesla, but the rotation angle of the beam will be nearly doubled from that of $5.3 \mathrm{GeV}$. Compensation design and adjustment will be significantly more critical than at higher energies.

Experiment background: The background hit rates and energy deposition have been modeled including synchrotron radiation, coulomb and bremstrahlung beamgas scattering, and Touschek scattering and found to be less than $5 \%$ of present values (at $5.3 \mathrm{GeV}$ ).

Beam-beam effects: A strong-strong beam-beam simulation code [10] run with no free parameters has reproduced measured CESR luminosity at $5.3 \mathrm{GeV}$. This same code has confirmed the estimated luminosity given in Table 1, albeit with different operating points in the tune plane because of a change in synchrotron frequency.

A parameter list for CESR-c operation is presented in the table below.

Table 1: CESR-c Parameters

\begin{tabular}{|l|c|c|c|c|}
\hline $\mathbf{E}_{\mathbf{0}}[\mathrm{GeV}]$ & $\mathbf{1 . 5 5}$ & $\mathbf{1 . 8 8}$ & $\mathbf{2 . 5}$ & $\mathbf{5 . 3}$ \\
\hline Luminosity & 150 & 300 & 500 & 1250 \\
{$\left[\div 10^{30} \mathrm{~cm}^{-2}\right.$-sec $\left.^{-1}\right]$} & 150 & & \\
$\mathrm{i}_{\mathrm{b}}\left[\mathrm{mA} / \mathrm{bunch}^{-}\right.$ & 2.8 & 4.0 & 5.1 & 8.2 \\
$\mathrm{I}_{\mathrm{beam}}[\mathrm{mA} / \mathrm{beam}]$ & 130 & 180 & 230 & 360 \\
$\xi_{\mathrm{y}}$ & 0.035 & 0.04 & 0.04 & .06 \\
$\xi_{\mathrm{x}}$ & 0.028 & 0.036 & 0.034 & .028 \\
$\sigma_{\mathrm{E}} / \mathrm{E}_{0}\left[\mathrm{x} 10^{3}\right]$ & 0.75 & 0.81 & 0.79 & 0.67 \\
$\tau_{\mathrm{x}, \mathrm{y}}[\mathrm{ms}]$ & 69 & 55 & 52 & 22 \\
$\mathrm{~B}_{\mathrm{W}}[\mathrm{Tesla}]$ & 2.1 & 2.1 & 1.75 & 0 \\
$\beta_{\mathrm{y}}^{*}[\mathrm{~cm}]$ & 1.0 & 1.0 & 1.0 & 1.8 \\
$\varepsilon_{\mathrm{x}}[\mathrm{nm}-\mathrm{rad}]$ & 230 & 220 & 215 & 205 \\
\hline
\end{tabular}

All configurations use 45 bunches/beam in 9 trains of 5 bunches and a horizontal crossing angle at the interaction point of $\pm 2.5-3.3 \mathrm{mr}$. $5.3 \mathrm{GeV}$ data are measured

\section{CONCLUSION}

The conversion of CESR to high luminosity operation over the energy range of 1.5 to $5.6 \mathrm{GeV}$ is a challenging but tractable accelerator physics and engineering project. Many of the issues, particularly those related to the wiggler, are common with other low energy colliders and damping rings. We expect to run with a luminosity of $3 \times 10^{32} \mathrm{~cm}^{-2}-\mathrm{sec}^{-1}$ at $1.9 \mathrm{GeV}$.

\section{REFERENCES}

[1] D.L. Rubin et. al., PAC'01 RPPH121, RPPH122

[2] R. H. Helm et al., PAC'73, IEEE NS-20 p. 900

[3] A. Mikhailichenko, PAC'01, RPPH320.

[4] J. Corbett, Y Nosochkov, PAC'99 p. 2358,

[5] J. Safranek et al., EPAC'00, p. 295,

[6] D. Sagan, "BMAD” dcs@ @esr10.lns.cornell.edu

[7] S. Belomestynkh, et al., PAC'01 MPPH124

[8] S. Henderson, J. Welch et. al. PAC'99 p. 3221

[9] M. Billing et al., PAC'01 TPAH005

[10] E. Anderson, J. Rogers, Cornell Note CBN97-33 